
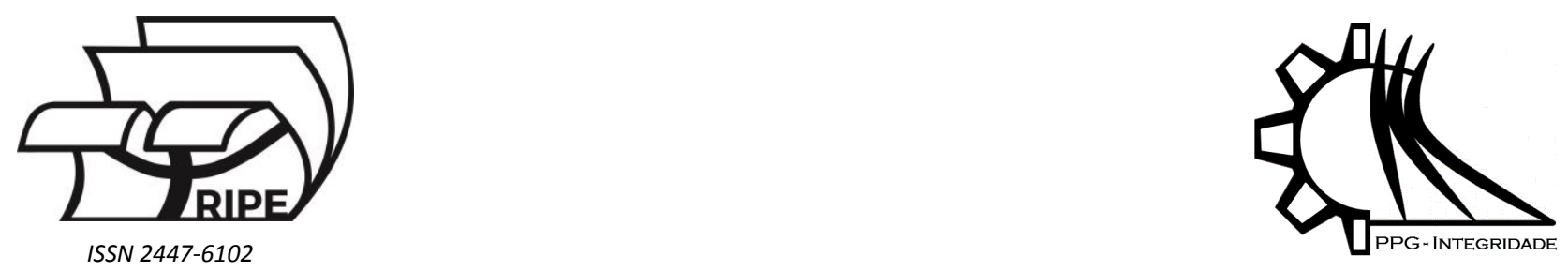

Article

\title{
Identificação de setores com necessidade de dragagem na hidrovia da Lagoa Mirim - RS, Brasil
}

\author{
Bendô, A.R.R. ${ }^{1, *}$, Foster, A.${ }^{2}$, Trombetta, T.B. ${ }^{1}$, Oleinik, P.H. ${ }^{1}$, Kirinus, E.P. ${ }^{1}$, Costi, J. ${ }^{2}$ e Marques, W.C. ${ }^{2}$ \\ 1 Escola de Engenharia - PPGEO, Universidade Federal do Rio Grande, Av Itália s/n km 8, Rio Grande CEP 96201-900, RS, Brasil; \\ rayltonbendo@gmail.com, thaisa bt@hotmail.com, phe.h.o1@gmail.com, ekirinus@gmail.com \\ 2 Instituto de Matemática, Estatística e Física - IMEF, Universidade Federal do Rio Grande, Av Itália s/n km 8, Rio Grande CEP \\ 96201-900, RS, Brasil; aldairforster@gmail.com, ju.costi@gmail.com, wilianmarques@furg.br \\ * Correspondence: rayltonbendo@gmail.com; Tel.: (53) 98163-6362
}

Received: 14/12/2018; Accepted: 05/01/2019; Published: 06/02/2019

\begin{abstract}
Resumo: $\mathrm{O}$ transporte hidroviário está entre os meios de transporte mais antigos e usados no mundo, além disso, quando comparado com outros modais de transporte mais utilizados no Brasil, apresenta uma melhor eficiência energética e acarreta menores impactos sociais e ambientais. Atualmente se tem discutido sobre a ampliação do potencial de transporte de cargas entre o Brasil e o Uruguai com a reabertura da Hidrovia da Lagoa Mirim. Portanto, conhecer a variabilidade natural do nível das águas desse ambiente é de extrema importância social, econômica e ambiental, uma vez que, a hidrodinâmica do corpo de água afeta diretamente as condições de navegabilidade, padrões de deposição de sedimentos e as operações de dragagem. No presente trabalho, investigamos a hidrodinâmica da Lagoa Mirim através de modelagem numérica, o foco principal é analisar as séries temporais de profundidade desse corpo lagunar e apontar os locais a serem dragados. Concluiu-se que a variabilidade do nível dessa lagoa é governada principalmente pela descarga dos rios em escalas intra-sazonais e anuais, e pelos ventos em escalas sinóticas de tempo. Através dos resultados obtidos, foi possível inferir que as regiões que necessitam de dragagem estão na parte norte, um pequeno trecho central e no extremo sul da Lagoa Mirim.
\end{abstract}

Palavras-chave: Dragagem, Lagoa Mirim, Hidrovias, Modelagem Hidrodinâmica, TELEMAC2D.

\section{Identification of in-need dredging sectors in the Mirim lagoon Waterway - RS, Brazil}

\begin{abstract}
Water transportation is one of the oldest and most used means of transportation in the world. In addition, when compared to other transportation modals most used in Brazil, it has a better energy efficiency, and lower social and environmental impacts. Nowadays there has been discussion about the expansion of the cargo transportation potential between Brazil and Uruguay with the reopening of the Mirim Lagoon Waterway. Therefore, knowing the natural variability of the water level of this environment is of extreme social, economic, and environmental importance, since the hydrodynamics of the water body directly affects the navigability conditions, sediment deposition patterns, and dredging operations. In the present work, we investigated the hydrodynamics of Mirim Lagoon through numerical modeling. The main focus is to analyze the depth time series of this lagoon body, and to point out the places to be dredged. It was concluded that the variability of the level of this lagoon is governed mainly by the discharge of the rivers in intra-seasonal and annual time scales, and by the winds in synoptic time scales. From the results obtained, it was possible to infer that the regions that require dredging are in the northern part, a small central stretch, and in the extreme south of Mirim Lagoon.
\end{abstract}

Keywords: Dredging, Mirim Lagoon, Waterways, Hydrodynamic Modeling, TELEMAC2D. 


\section{INTRODUÇÃO}

As redes hidroviárias estão entre os meios de transporte mais antigos e usados em todo mundo, exercendo importante função de movimentação de capitais de uma nação (Mihic, Golusin, \& Mihajlovic, 2011; Willems, Busscher, Woltjer, \& Arts, 2018). Segundo Santana e Tachibana (2004), ao ser comparado com outros modais de transporte, principalmente os terrestres rodoviário e ferroviário, o transporte aquaviário apresenta um excelente rendimento energético e pode ser considerado como aquele que talvez cause menores impactos sociais e ambientais (Azambuja, 2005; Garcia, Salort, \& Nääs, 2015). Além disso, o uso de vias navegáveis interiores está se tornando ainda mais relevante quando consideramos sua crescente integração com outros modos de transporte dentro de esquemas de redes de fretes multimodais (Lowe, 2005; Ruiz, Shi, \& Voß, 2018).

A Hidrovia do MERCOSUL (HM) se localiza no extremo sul do Brasil e a leste do Uruguai, é constituída, dentre outros, pelo Rio Cebollati, Tacuari, Jaguarão, atravessa a Lagoa Mirim (LM) e se conecta à Lagoa dos Patos (LP) através do canal de São Gonçalo (SG), um canal natural com 78 quilômetros de extensão (Hirata, Möller, \& Mata, 2010; Oliveira, Fernandes, Möller, \& Collares, 2015). De acordo com o Decreto legislativo no 546, de 2002, uma vez implantado, esse sistema viário flúvio-lacustre ampliará o comércio entre os dois países, pois a via proporcionará que os produtos uruguaios cheguem até o porto de Rio Grande, com saída através da cabotagem para a costa brasileira, ou mesmo para navegações de longo curso em mercados internacionais, trazendo reflexos positivos para o desenvolvimento da região da LM e de sua área de influência.

Atualmente, tem se discutido sobre a reabertura da HM e o aumento do uso industrial das águas da LM. De acordo com Costi, Marques, Kirinus, Duarte, e Arigony-Neto (2018) entender a variabilidade do nível de água desse ambiente é de grande importância social, econômica e ambiental, visto que, a hidrodinâmica do corpo de água afeta diretamente a qualidade e disponibilidade deste recurso, modifica as condições de navegabilidade, o tempo de residência dos poluentes, os padrões de deposições de sedimentos e as operações de dragagem (Legesse \& Vallet-Coulomb, 2004; Munar et al., 2018). Entretanto a hidrodinâmica da LM tem sido pouco estudada (Hirata et al., 2010; Oliveira et al., 2015; Costi et al., 2018; Munar et al., 2018).

O objetivo desse trabalho é estudar a variabilidade dos níveis d'água em resposta à descarga dos rios e à ação dos ventos na LM, utilizando o modelo hidrodinâmico TELEMAC-2D. O foco principal é analisar as séries temporais de profundidade da LM e apontar os locais que necessitam de dragagem com intuito de garantir uma condição segura à navegação. Atualmente o calado máximo da LM é de $2,5 \mathrm{~m}$ e a coluna d'água necessária para atender de forma segura a navegabilidade é de $3 \mathrm{~m}$, a proposta é uniformizar a HM do trecho dentro da LM com um calado de 5,2 m com nível mínimo de água de $6 \mathrm{~m}$ (Calado e profundidade referentes ao canal do Porto de Pelotas).

\section{1 Área de estudo}

A LM é uma lagoa costeira que possui $180 \mathrm{~km}$ de extensão ao longo de seu eixo central, orientado para nordeste-sudoeste. Está localizada no extremo sul do Brasil, entre as latitudes $32^{\circ} 09^{\prime}$ e $33^{\circ} 32^{\prime} \mathrm{S}$ e longitudes $52^{\circ} 35^{\prime}$ e $53^{\circ} 58^{\prime} \mathrm{W}$, é o terceiro maior corpo d'água da América do Sul, com $3748 \mathrm{~km}$., dos quais um terço está localizado em território uruguaio e dois terços em território brasileiro. A Lagoa Mirim se conecta à Lagoa dos Patos através do canal de São Gonçalo formando o maior sistema lagunar da América do Sul, conforme a Fig. 1 (Santos, Baisch, Lima, \& Silva Filho, 2004).

A região possui clima subtropical, com uma temperatura média de $16^{\circ} \mathrm{C}$ e precipitação oscilando cerca de 1.200 $\mathrm{mm}$ a $2.000 \mathrm{~mm}$ em média anualmente (Santos et al., 2004). A superfície da bacia de drenagem possui $58.400 \mathrm{~km}$, dos quais $32.704 \mathrm{~km}$ estão no Uruguai e $25.696 \mathrm{~km}$ no Brasil. A bacia tem seis sub-bacias principais, a maior do lado brasileiro é a do rio Jaguarão, e a do Cebollati é a maior do lado uruguaio, o volume da lagoa pode atingir $17 \mathrm{~km}$, dependendo das condições hidrológicas e do fluxo de saída (Munar et al., 2018).

As taxas de precipitação observadas na região sofrem impactos importantes do fenômeno oceânico El Niño (Grimm \& Saboia, 2015), essas taxas controlam diretamente a descarga dos rios na região, onde as maiores descargas ocorrem no inverno e na primavera (Mechoso \& Iribarren, 1992).

A direção e intensidade dos ventos são altamente variáveis durante o ano na região. A direção predominante é de nordeste, mas durante o outono e inverno há um acréscimo na incidência do quadrante sul em contraste com o que ocorre no verão e primavera (Spagnoli et al., 2002). Os ventos prevalecentes de nordeste são relacionados da circulação atmosférica anticiclônica sobre o Oceano Atlântico, enquanto que os ventos sul e sudeste ocorrem devido a passagem de frentes frias e ciclones extras tropicais (Parise, Calliari, \& Krusche, 2009). 


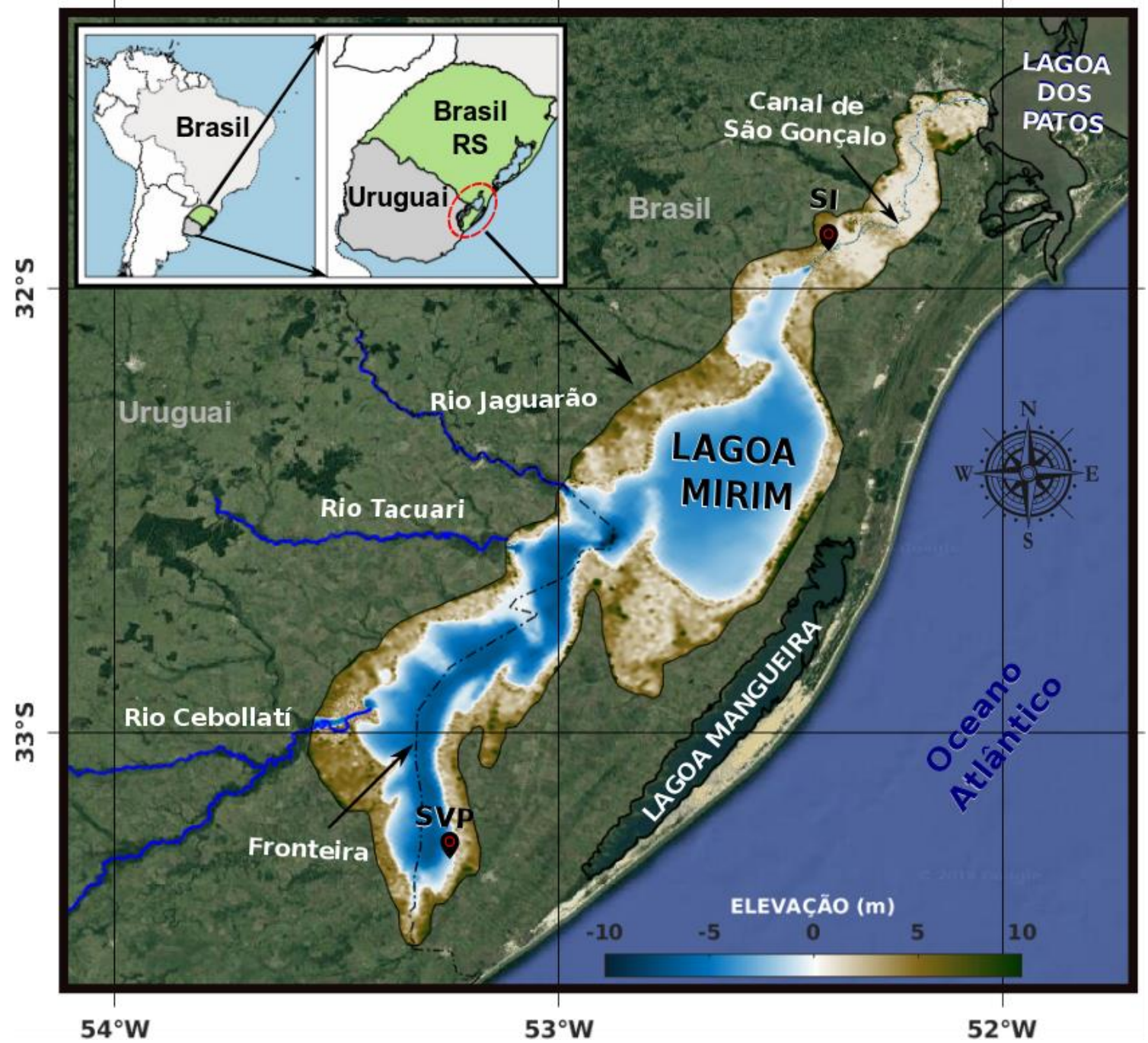

Figura 1. A figura mostra a elevação da Lagoa Mirim, adjacências e sua localização na América do Sul. Os valores negativos correspondem a batimetria e os positivos à topografia da planície de inundação

\section{METODOLOGIA}

\subsection{Modelo Hidrodinâmico}

O presente estudo foi realizado utilizando o módulo hidrodinâmico TELEMAC-2D, pertencente ao sistema Open TELEMAC-MASCARET (www.opentelemac.org). Esse sistema é baseado na técnica dos Elementos Finitos (Hervouet, 2000, 2007).

O modelo TELEMAC-2D resolve as equações de Saint-Venant para escoamentos em superfície livre aceitando a hipótese de equilíbrio hidrostático. Além disso, o mesmo considera a evolução da superfície livre em função do tempo e utiliza as equações de advecção-difusão para o transporte das concentrações dos traçadores (salinidade, temperatura ou sedimentos em suspensão) (Hervouet, 2007). A turbulência foi parametrizada com o modelo $k-\varepsilon$, que resolve as equações de transporte e dissipação da energia cinemática turbulenta. Esse modelo é amplamente usado em lagoas costeiras e na plataforma continental sul do Brasil (Kirinus \& Marques, 2015; Costi et al., 2018; Marques et al., 2017).

A simulação foi realizada por cinco anos, começando de 01 de Janeiro de 2000 a 31 de Dezembro de 2004, o modelo foi forçado pela ação dos ventos e das descargas dos rios e a malha foi elaborada a fim de garantir a reprodução da complexa batimetria dos canais e da linha de costa e a discretização de seus elementos variam de 30 a $1.000 \mathrm{~m}$. Os dados batimétricos da LM e do Canal de São Gonçalo foram obtidos a partir de cartas náuticas levantadas pela Diretoria de Hidrografia e Navegação (DHN). Os dados topográficos da planície de inundação foram obtidos a 
partir do modelo digital de elevação SRTM (Shuttle Radar Topography Mission) (Farr et al., 2007) e através do mapeamento de zonas úmidas utilizando imagens do sensor RADAR de Abertura Sintética ERS2 (European Remote Sensing Satellite) (Costi, Oleinik, Monteiro, Marques, \& Arigony-Neto, 2017).

Os rios Jaguarão, Tacuarí, Cebollatí e a foz do canal de São Gonçalo (ver na Fig. 1), considerados no domínio computacional como contornos líquidos abertos, foram forçados com séries temporais com resoluções diárias, medida pela Agência Nacional de Águas (ANA), e nível de água registrada na foz do Canal de São Gonçalo. Esses dados estão disponíveis no site (http://hidroweb.ana.gov.br). A condição superficial foi forçada pelas velocidades dos ventos, pressão atmosférica e temperatura do ar do conjunto de dados de reanálise do projeto ERA-Interim, com resolução temporal de seis horas (Dee et al., 2011).

\subsection{Determinação dos níveis de referência para dragagem}

Para identificação das áreas a serem dragadas foi analisado as séries temporais de níveis d'água, e pode-se gerar uma informação conhecida como curva de permanência, que significa obter a percentagem de tempo (ou permanência) no qual determinado evento é igualado ou superado sobre todo o período histórico investigado (Quimpo, Alejandrino, \& McNally, 1983; Vogel \& Fennessey, 1994). Estudos com esta conotação têm várias importâncias práticas como, por exemplo, na determinação de um nível mínimo de um curso d'água que permita estabelecer critérios para segurança da navegação (Jehng-Jung \& Bau, 1996).

Quando fala-se de gestão de recursos hídricos e legislação ambiental, o valor prático que se costuma extrair da curva de permanência é o percentual de $90 \%$ correspondente a um nível existente no curso d'água em $90 \%$ do tempo. Pela curva, observa-se que o risco assumido do nível d'água ficar abaixo do valor projetado é de $10 \%$.

Para o atual estudo foi escolhido o nível que garanta a navegabilidade segura na LM em $90 \%$ ou mais do tempo, segundo Vogel e Fennessey (1994) o percentual pode ser estimado a partir de uma função empírica de percentis (probabilidades acumuladas), baseado na definição de uma posição de plotagem. Este critério foi escolhido com objetivo de obter o maior aproveitamento do recurso para a movimentação de cargas e redução de custos excessivos com dragagens.

Estabelecido o nível de permanência, foram identificados os locais onde as profundidades são menores do que 3 e 6 metros (colunas d'água referentes ao calados de 2,5m e 5,2m). Para melhorar a precisão da análise, o trecho da HM dentro da LM foi dividido em sete subtrechos (denominados como T1, T2, T3, T4, T5, T6 e T7) (Fig. 3).

\section{RESULTADOS}

\subsection{Avaliação das Seções Lagunares}

A análise do comportamento hidrodinâmico está focada na influência dos ventos e das descargas dos rios sobre a variação dos níveis de água da LM durante o período de simulação. A hidrodinâmica é discutida em termos de valores mínimos, médios e tendências das séries temporais de profundidade de água da LM. Desse modo, a investigação está baseada na identificação dos locais da HM onde os níveis de permanência são inferiores às profundidades necessárias para navegação segura.

Na Fig. 2, pode-se observar a variação da altura de água no corpo lagunar da Lagoa Mirim, onde valores mínimos, médios e o desvio padrão são apresentados. Os resultados da simulação foram reduzidos espacialmente para mostrar apenas a posição da HM dentro da LM, facilitando a sua observação.

Em termos de padrões mínimos (Fig. 2A), o parâmetro da profundidade da água varia de acordo com a extensão da Lagoa Mirim de forma que seus valores mais elevados estão na borda sudeste e o oposto é observado em direção ao Canal São Gonçalo. Ainda neste sentido, é possível observar locais no centro da LM em que o nível de d'água apresenta valores abaixo de $3 \mathrm{~m}$ (tons azuis na Fig. 2A.).

Do mesmo modo, os valores médios apresentaram o mesmo padrão de distribuição ao longo do corpo lagunar, na parte norte são encontrando os menores valores, com cerca de $6 \mathrm{~m}$ (ver na Fig. 2B.). Por outro lado, a parte sul da LM mostra profundidades acima de $9 \mathrm{~m}$, com exceção de um trecho ao centro da LM, onde são encontradas profundidades médias em torno de $5 \mathrm{~m}$.

Na Fig.2C o desvio padrão da profundidade da água apresenta valores mínimos de 0,90 m ao norte e 0,95 $\mathrm{m}$ ao sul, demostrando variabilidades de quase $1 \mathrm{~m}$ em torno da média ao longo do corpo lagunar durante o período de 5 anos de simulação. 


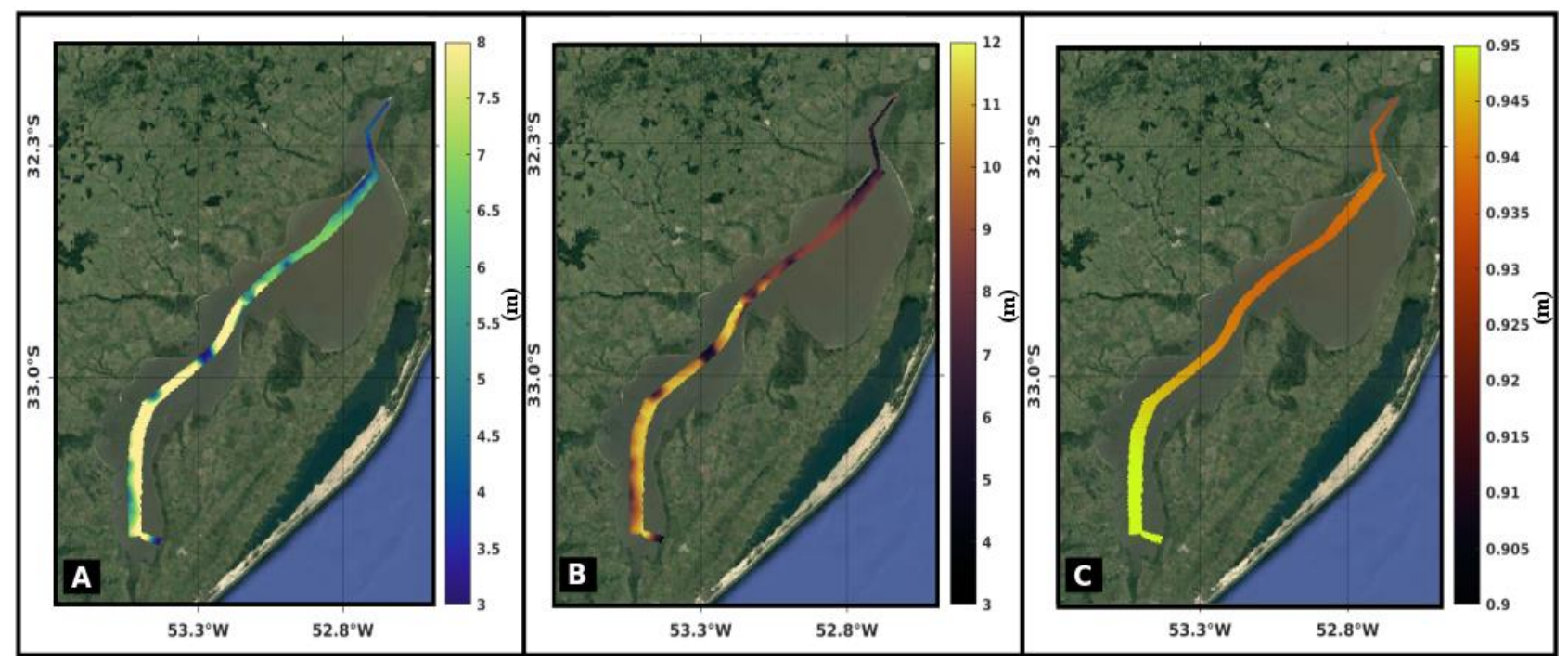

Figura 2. As figuras A, B e C são respectivamente a mínima, média e o desvio padrão (em metros) das séries temporais de profundidade de água do trecho da HM dentro da LM.

Após a realização do calculo do nível de permanência para todo o trecho da HM, obteve-se a altura da coluna d'água relativa ao nível de permanência. Para melhorar a visualização, a hidrovia (Fig. 3) foi dividida em sete subtrechos, dois ao norte, denominados de T1 e T2, na parte central T3, T4 e T5 e ao sul T6 eT7.

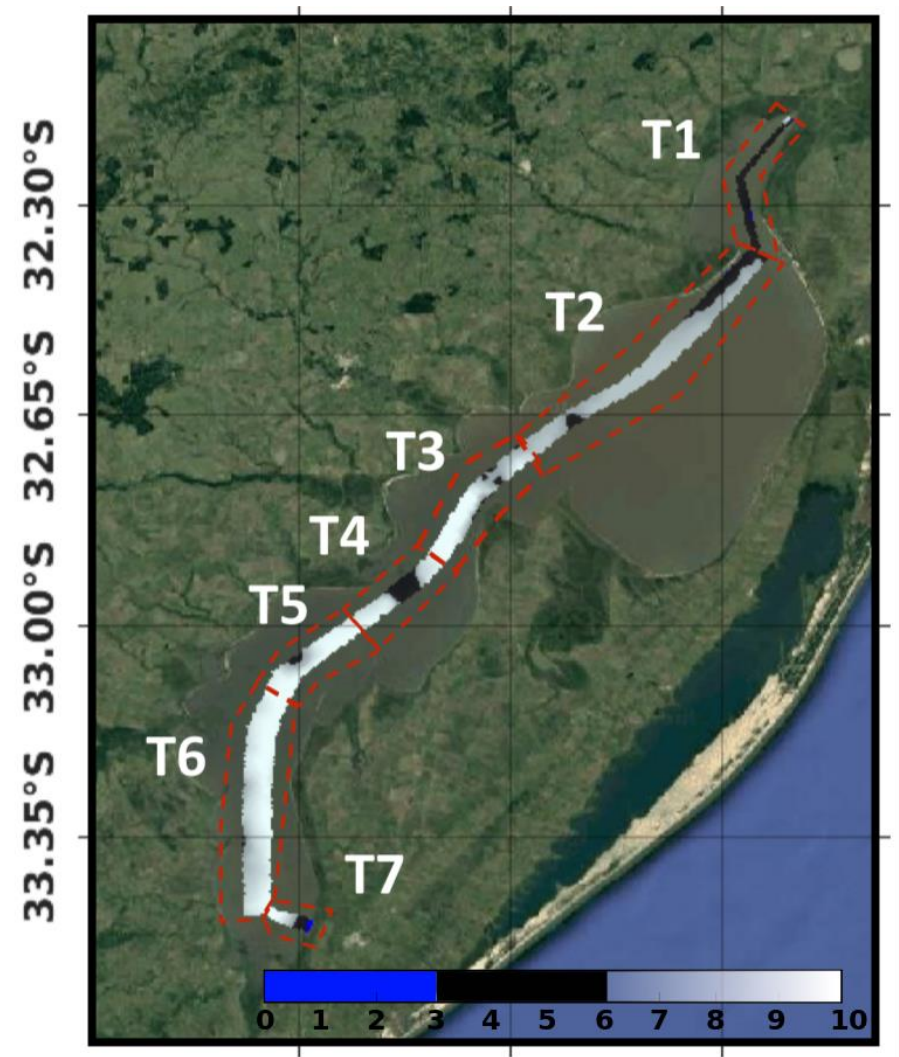

$53.45^{\circ} \mathrm{W} 53.10^{\circ} \mathrm{W} 52.75^{\circ} \mathrm{W}$

Figura 3. Figura $\mathrm{A}$ mostra as profundidades da $\mathrm{HM}$ em relação ao nível de permanência de todo o trecho da $\mathrm{HM}$ e suas subdivisões.

Os resultados dos níveis de permanência da LM são mostrados na Fig. 3, destacados nas cores azul e preto os locais com profundidades menores que $3 \mathrm{~m}$ e $6 \mathrm{~m}$, respectivamente. Vale ressaltar que os locais mais críticos para a 
navegação são aqueles ao norte da LM (T1) e na região do porto de SVP (T7). Entretanto, vale destacar que em uma zona rasa na parte central da LM (T4) será necessário realizar atividades de dragagem para garantir a segurança na navegação com coluna d'água mínima de $6 \mathrm{~m}$.

Nos demais trechos da hidrovia, pode-se afirmar que poderá haver movimentação das cargas de forma segura, como mostra os resultados da simulação (níveis de permanência em tons de cinza). Como foi mostrando na Fig.3, a região T7 é a mais preocupante, assumindo valores menores que $3 \mathrm{~m}$, devido as baixas profundidades da batimetria.

Através dos resultados encontrados na Fig. 3, foi possível concluir que os trechos abaixo das cotas de projeto são os T1, T4 e T7. Pode-se destacar ainda que, os trechos T1 e T4 não seriam dragados caso houvesse tráfego de embarcações que necessitassem de no mínimo $6 \mathrm{~m}$ de profundidade. Já na região próxima ao porto de SVP, as atividades de dragagem devem ser necessariamente realizadas.

De forma a corroborar esses resultados, os processos referentes aos níveis de água abaixo de $6 \mathrm{~m}$ na LM foram avaliados em formato de porcentagem de tempo e sua relação com a quantidade de dias em que os trechos da lagoa assumiram valores de profundidades menores do que $3 \mathrm{~m}$ e $6 \mathrm{~m}$ (presentes na tabela 1).

Tabela 1. Número de dias e porcentagem do tempo que os subtrechos da HM ficam acima e abaixo do nível de água estabelecido.

\begin{tabular}{|c|c|c|c|c|c|c|c|c|}
\hline \multirow{2}{*}{$\begin{array}{c}\text { PERÍODO DE } \\
\text { SIMULAÇÃo } \\
2000-2004\end{array}$} & \multicolumn{4}{|c|}{$\begin{array}{c}\text { Tempo abaixo } \\
\text { do nível mínimo }\end{array}$} & \multicolumn{2}{|c|}{$\begin{array}{r}\text { Tempo acima } \\
\text { nível mínimo }\end{array}$} & $\begin{array}{r}\text { Tempo abaixo } \\
\text { nível mínimo }\end{array}$ & \multicolumn{3}{|c|}{$\begin{array}{c}\text { Tempo acima } \\
\text { nível mínimo }\end{array}$} \\
\cline { 2 - 10 } & \% & Dias & $\%$ & Dias & $\%$ & Dias & $\%$ & Dias \\
\hline T1 & 1,5 & 27,40 & 98,5 & 1799,60 & 43,77 & 799,68 & 56,23 & 1027,32 \\
\hline T2 & 0,1 & 18,27 & 99,90 & 1807,73 & 8,30 & 151,60 & 91,70 & 1675,40 \\
\hline T3 & 0,01 & 1,83 & 99,99 & 1825,27 & 2,50 & 45,67 & 97,5 & 1781,33 \\
\hline T4 & 0,03 & 5,48 & 99,97 & 1821,52 & 14,51 & 265,10 & 85,49 & 1561,90 \\
\hline T5 & 0,0 & 0,0 & 100 & 1827 & 1,13 & 20,64 & 98,87 & 1806,36 \\
\hline T6 & 0,0 & 0,0 & 100 & 1827 & 0,25 & 4,57 & 99,75 & 1822,25 \\
\hline T7 & 7,12 & 131,54 & 92,89 & 1695,46 & 25,53 & 466,43 & 74,47 & 1360,57 \\
\hline
\end{tabular}

Na tabela 1, as duas cotas de trabalho $(3 \mathrm{~m} \mathrm{e} 6 \mathrm{~m}$ ) estão apresentadas para cada subtrecho. No caso da profundidade igual à $3 \mathrm{~m}$, em quase todos os subtrechos temos aproximadamente $100 \%$ de água acima deste valores, exceto para o trecho T7. Por outro lado para o valor de $6 \mathrm{~m}$, os subtrechos T2, T3, T5 e T6 demonstram nível acima de $6 \mathrm{~m}$ por mais de $90 \%$ do tempo simulado.

Entretanto os subtrecho T1, T4 e T7 apresentaram 56,23\%, 85,49\% e 74,47\% acima da cota de $6 \mathrm{~m}$, respectivamente. Este resultado significa que, em relação aos 5 anos de simulação, a região ao norte da LM (T1) permaneceu 799 dias locais com profundidades abaixo do nível desejado. Além disso, foi observado para subtrecho T4 e T7 ficaram com níveis inferiores a $3 \mathrm{~m}$ e $6 \mathrm{~m}$ durante, respectivamente, 265 e 466 dias, indicando que esses locais devem ser dragados para atender aos critérios estabelecidos anteriormente.

\subsection{Fatores Meteo-Oceanográficos}

O clima de ventos e correntes referentes aos cinco anos de simulação é apresentado na Fig.4 na forma de rosa dos ventos e correntes para as extremidades norte (P1), sul (P3) e região central (P2). A rosa de ventos nos mostra que a predominância dos ventos são de nordeste, principalmente durante o verão e primavera, esse fenômeno está relacionado com a circulação atmosférica anticiclônica sobre o Oceano Atlântico Sul, entretanto, durante o outono e inverno há uma inversão da direção dominante, com as passagens durante a passagem de frentes frias e ciclones extras tropicais (Stech \& Lorenzetti, 1992).

A direção e intensidade das correntes se comportam de forma diferente ao longo do comprimento LM. No ponto mais ao norte (P1), o sentido do escoamento predominante é nordeste, fluindo contra os ventos, entretanto, tal sentido pode se inverter em algumas épocas do ano por períodos curtos de tempo, isso ocorre pelo enfraquecimento da descarga fluvial e incidência de ventos de nordeste, que podem ocorrer, principalmente, no verão e primavera. Dessa forma, percebe-se que a direção e intensidade das correntes são influenciadas principalmente pela descarga dos rios. 


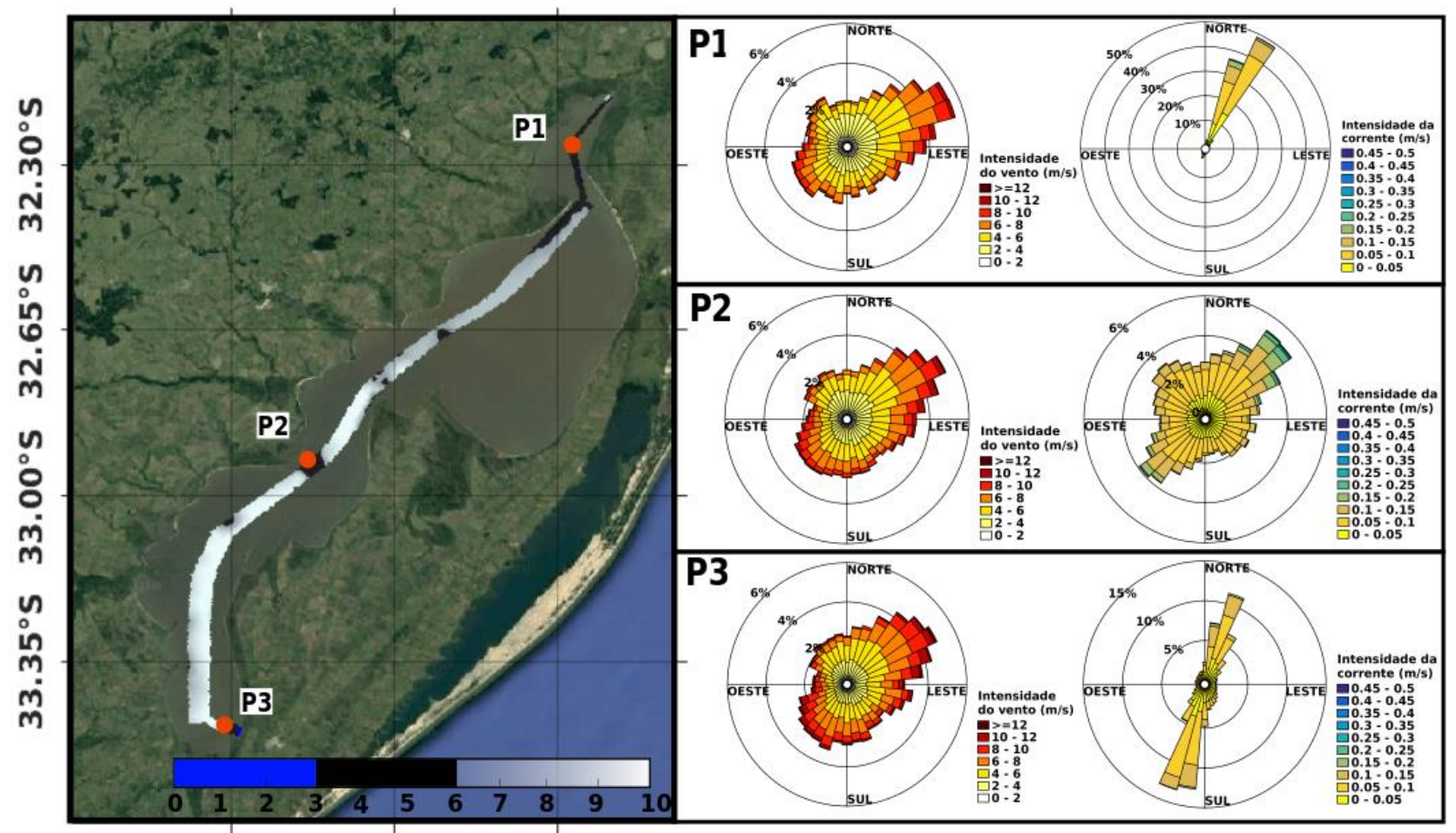

$53.45^{\circ} \mathrm{W} 53.10^{\circ} \mathrm{W} 52.75^{\circ} \mathrm{W}$

Figura 4. A figura mostra as profundidades da HM em relação ao nível de permanência da HM, os pontos P1, P2 e P3 representados pelos pontos em vermelho e suas respectivas rosas de ventos e correntes.

No ponto P2 as correntes tem grande variabilidade em sua direção e intensidade, isso pode ser causado por estar localizado nas proximidades das desembocaduras dos rios Cebollati e Tacuari, que geram vórtices na região, causando essa maior variabilidade de direção. A principal forçante que atua na circulação é a descarga dos rios, seguida das ações dos ventos.

De forma oposta aos pontos P1 e P2, no ponto P3, por estar próximo a margem da LM, as correntes são paralelas a linha de costa como se pode observar na Fig.4. Nas estações menos chuvosas o vento atua com principal forçante, porém, o contrário ocorre nas estações chuvosas quando as descargas dos rios atuam com maior intensidade na direção e velocidade das correntes.

As séries temporais de nível dos pontos P1, P2 e P3 são apresentadas na Fig.5. Os níveis de água mais altos são observados no final de outono até o início da primavera, demonstrando um padrão sazonal de precipitação da região em estudo (Hirata et al., 2010; Costi et al., 2018). De forma semelhante, Moller, Lorenzzentti, Stech, e Mata (1996) afirmaram que os picos de descarga dos rios da bacia de drenagem da Lagoa dos Patos ocorrem no inverno e primavera. Assim, a LM manifesta padrões sazonais semelhantes aos da bacia vizinha.

A Fig. 5A, mostra os níveis em cada um dos pontos de análise. As séries temporais de nível evidenciam que os pontos, embora estejam localizados nos extremos da LM, apresentam um mesmo padrão de variabilidade temporal. As análises de ondaletas feitas por Costi et al. (2018) sugerem que a LM se comporta como um sistema fechado e seu nível é dominado pela descarga dos rios em escalas de tempo intra-sazonais, sazonais e anuais, como pode-se observar na Fig. 5C. Entretanto, os ventos promovem oscilações no nível em menores escalas de tempo Fig. 5B. 


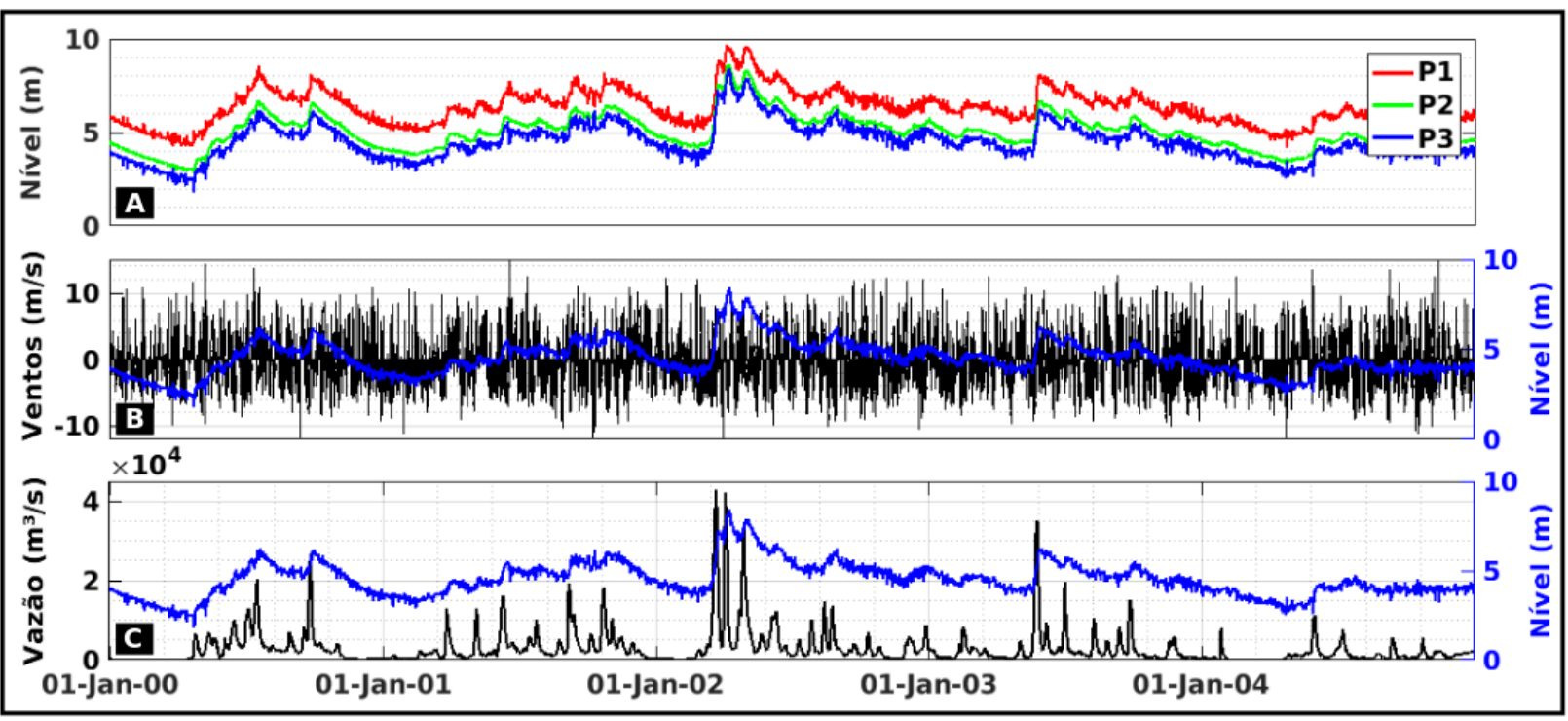

Figura 5. As figuras A, B e C mostram respectivamente séries históricas de nível em P1, P2 e P3, a comparação entre os ventos e o nível em P3 e por último a vazão versus nível no mesmo ponto.

\section{Conclusões}

No presente trabalho, foram analisados os resultados de cinco anos de simulação hidrodinâmica bidimensional da Lagoa Mirim. A variabilidade dos níveis de água dessa lagoa é dominada, principalmente, pela descarga fluvial, seguida da ação dos ventos em diferentes escalas de influência. A contribuição dos ventos é notada nas correntes na região norte, provocando vórtices que modificam a direção das correntes próxima ao porto de Santa Vitória do Palmar.

A dinâmica forçada pela descarga fluvial dos rios altera os padrões hidrodinâmicos nos locais de interesse para dragagem. Portanto, podemos concluir que nos trechos T1, T2 e T4, não há necessidade de aprofundamento do canal para o tráfego de embarcações com calado de $2,5 \mathrm{~m}$. Contudo, será necessário dragar esses locais caso haja tráfego de embarcações que necessitem de no mínimo $6 \mathrm{~m}$ de coluna d'água. Já o trecho sete (T7), região próxima ao porto de Santa Vitória do Palmar, necessitará de constantes atividades de dragagem para garantir condições de navegabilidade na região.

Agradecimentos: Os autores agradecem à Coordenação de Aperfeiçoamento de Pessoal de Nível Superior (CAPES) pela concessão das bolsas de estudo, ao Conselho Nacional de Desenvolvimento Científico e Tecnológico (CNPq) pelo contrato 304227/2016-1, à Fundação de Amparo à Pesquisa do Estado do Rio Grande do Sul (FAPERGS) pelo contrato 17/2551-0001 159-7 e ao Centro Nacional de Supercomputação (CESUP), da Universidade Federal do Rio Grande do Sul (UFRGS), que auxiliaram o desenvolvimento deste trabalho. Agrade cem também à Agência Nacional de Águas (ANA), à NOAA e ao HYCOM pelos dados hidrológicos, oceanográficos e meteorológicos utilizados nas condições de contorno dos modelos, ao consórcio Open TELEMAC-MASCARET por disponibilizar o sistema TELEMAC gratuitamente e ao Laboratório Nacional de Com putação Científica (LNCC) pela disponibilização de uso do Supercomputador Santos Dumont.

\section{Referências}

1. Azambuja, J. L. F., \& Luiz, J. (2005). Hidrovia da Lagoa Mirim: Um marco de desenvolvimento nos caminhos do Mercosul. Escola de Engenharia. Universidade Federal do Rio Grande do Sul. Porto Alegre, Brasil.

2. Costi, J., Marques, W. C., de Paula Kirinus, E., de Freitas Duarte, R., \& Arigony-Neto, J. (2018). Water level variability of the Mirim-São Gonçalo system, a large, subtropical, semi-enclosed coastal complex. Advances in Water Resources, 117, 75-86.

3. Costi, J., Oleinik, P. H., Monteiro, C. B., Marques, W. C., \& Arigony-Neto, J. (2016). An Automated Structure for Acquiring and Processing Sentinel-1 Data and its Applicability for Coastal Studies. In Defect and Diffusion Forum (Vol. 372, pp. 122-131). Trans Tech Publications. 
4. Dee, D. P., Uppala, S. M., Simmons, A. J., Berrisford, P., Poli, P., Kobayashi, S., ... \& Bechtold, P. (2011). The ERAInterim reanalysis: Configuration and performance of the data assimilation system. Quarterly Journal of the royal meteorological society, 137(656), 553-597.

5. Farr, T. G., Rosen, P. A., Caro, E., Crippen, R., Duren, R., Hensley, S., ... \& Seal, D. (2007). The shuttle radar topography mission. Reviews of geophysics, 45(2).

6. Garcia, S., Vicens-Salort, E., \& Nääs, I. A. (2015). INVESTIMENTOS EM TRANSPORTE INTERMODAL NO BRASIL PODERIA BENEFICAR O CRESCIMENTO DO PIB/INVESTMENT IN INTERMODAL TRANSPORTATION IN BRAZIL COULD BENEFIT THE COUNTRY'S AGRIBUSINESS GDP GROWTH. Revista Brasileira de Engenharia de Biossistemas, 9(1), 90-98.

7. Grimm, A. M., \& Saboia, J. P. (2015). Interdecadal variability of the South American precipitation in the monsoon season. Journal of Climate, 28(2), 755-775.

8. Hervouet, J. M. (2000). TELEMAC modelling system: an overview. Hydrological Processes, 14(13), $2209-2210$.

9. Hervouet, J. M. (2007). Hydrodynamics of free surface flows: modelling with the finite element method. John Wiley \& Sons.

10. Hirata, F. E., Möller Junior, O. O., \& Mata, M. M. (2010). Regime shifts, trends and interannual variations of water level in Mirim Lagoon, southern Brazil.

11. Kao, J. J., \& Bau, S. F. (1996). Risk analysis for flow duration curve based seasonal discharge management programs. Water Research, 30(6), 1369-1376.

12. de Paula Kirinus, E., \& Marques, W. C. (2015). Viability of the application of marine current power generators in the south Brazilian shelf. Applied Energy, 155, 23-34.

13. Legesse, D., Vallet-Coulomb, C., \& Gasse, F. (2004). Analysis of the hydrological response of a tropical terminal lake, Lake Abiyata (Main Ethiopian Rift Valley) to changes in climate and human activities. Hydrological processes, 18(3), 487-504.

14. Lowe, D. (2005). Inland waterway, short-sea, and coastal shipping. Lowe D., Intermodal Freight Transport. Oxford: Elsevier Butterworth-Heinemann, 92-108.

15. Marques, W. C., Stringari, C. E., Kirinus, E. P., Möller Jr, O. O., Toldo Jr, E. E., \& Andrade, M. M. (2017). Numerical modeling of the Tramandaí beach oil spill, Brazil-Case study for January 2012 event. Applied Ocean Research, 65, 178-191.

16. Mechoso, C. R., \& Iribarren, G. P. (1992). Streamflow in southeastern South America and the southern oscillation. Journal of climate, 5(12), 1535-1539.

17. Mihic, S., Golusin, M., \& Mihajlovic, M. (2011). Policy and promotion of sustainable inland waterway transport in Europe-Danube River. Renewable and sustainable energy reviews, 15(4), 1801-1809.

18. Moller Jr, O. O., Stech, J., \& Mata, M. M. (1996). The Patos Lagoon summertime circulation and dynamics. Continental Shelf Research, 16(3), 335-351.

19. Munar, A. M., Cavalcanti, J. R., Bravo, J. M., Fan, F. M., da Motta-Marques, D., \& Fragoso Jr, C. R. (2018). Coupling large-scale hydrological and hydrodynamic modeling: Toward a better comprehension of watershed-shallow lake processes. Journal of hydrology, 564, 424-441.

20. Oliveira, H. A. D., Fernandes, E. H. L., Möller Junior, O. O., \& Collares, G. L. (2015). Processos hidrológicos e hidrodinâmicos da Lagoa Mirim.

21. Parise, C. K., Calliari, L. J., \& Krusche, N. (2009). Extreme storm surges in the south of Brazil: atmospheric conditions and shore erosion. Brazilian Journal of Oceanography, 57(3), 175-188.

22. Quimpo, R. G., Alejandrino, A. A., \& McNally, T. A. (1983). Regionalized flow duration for Philippines. Journal of Water Resources Planning and Management, 109(4), 320-330.

23. Lalla-Ruiz, E., Shi, X., \& Voß, S. (2016). The waterway ship scheduling problem. Transportation Research Part D: Transport and Environment.

24. Santana, W. A., \& Tachibana, T. I. (2010). Caracterização dos elementos de um projeto hidroviário, vantagens, aspectos e impactos ambientais para a proposição de metodologias técnico-ambientais para o desenvolvimento do transporte comercial de cargas nas hidrovias brasileiras. Engevista, 6(3).

25. Santos, I. R. D., Baisch, P. R. M., Lima, G. T. N. P. D., \& Silva-Filho, E. V. D. (2004). Nutrients in surface sediments of Mirim lagoon, Brazil-Uruguay border.

26. Spagnoli, F., Specchiulli, A., Scirocco, T., Carapella, G., Villani, P., Casolino, G., ... \& Franchi, M. (2002). The Lago di Varano: Hydrologic characteristics and sediment composition. Marine Ecology, 23, 384-394. 
27. Stech, J. L., \& Lorenzzetti, J. A. (1992). The response of the South Brazil Bight to the passage of wintertime cold fronts. Journal of Geophysical Research: Oceans, 97(C6), 9507-9520.

28. Vogel, R. M., \& Fennessey, N. M. (1994). Flow-duration curves. I: New interpretation and confidence intervals. Journal of Water Resources Planning and Management, 120(4), 485-504.

29. Willems, J. J., Busscher, T., Woltjer, J., \& Arts, J. (2018). Co-creating value through renewing waterway networks: A transaction-cost perspective. Journal of Transport Geography, 69, 26-35. 